Am J Perinatol. 2017 September ; 34(11): 1125-1130. doi:10.1055/s-0037-1604161.

\title{
Prophylactic Wound Vacuum Therapy after Cesarean Section to Prevent Wound Complications in the Obese Population: A Randomized Controlled Trial (the ProVac Study)
}

\author{
Kelly Ruhstaller, MD ${ }^{1}$, Katheryne L. Downes, PhD, MPH ${ }^{1}$, Suchitra Chandrasekaran, MD, \\ MSCE$^{2}$, Sindhu Srinivas, MD, MSCE ${ }^{1}$, and Celeste Durnwald, MD ${ }^{1}$ \\ ${ }^{1}$ Department of Obstetrics and Gynecology, Maternal Child Health Research Center, Perelman \\ School of Medicine, University of Pennsylvania, Philadelphia, Pennsylvania \\ ${ }^{2}$ Department of Obstetrics and Gynecology, University of Washington, Seattle, Washington
}

\begin{abstract}
Objective-The objective of this study was to perform a randomized controlled feasibility trial investigating negative pressure wound therapy (NPWT) system versus a standard postcesarean wound care (WC) on the development of a postoperative surgical site infection (SSI) and/or a wound dehiscence in obese women.
\end{abstract}

Study Design-This is a randomized controlled feasibility trial of obese women undergoing an unscheduled cesarean delivery. Women with an initial body mass index $\geq 30 \mathrm{~kg} / \mathrm{m}^{2}$ who were $\geq 4$ $\mathrm{cm}$ dilated were included. Women were assigned to either a NPWT or standard WC. The primary outcome was a composite of wound morbidity at 4 weeks postpartum including SSI and/or wound opening (clinicaltrials. gov, NCT02128997). Continuous variables were analyzed with $t$-test and Wilcoxon rank-sum tests and categorical variables with Fisher's exact test.

Results-Of 136 women randomized, 67 received NPWT and 69 received standard WC. The 4week follow-up rate was $88 \%$. Maternal clinical and surgical characteristics were similar between the groups. The prevalence of the composite wound morbidity outcome was not different between those with NPWT and standard WC (4.9 vs. $6.9 \% ; p=0.71)$.

Conclusion-Routine clinical use of a NPWT system after cesarean delivery did not result in a significant reduction of wound morbidity over standard WC.

\footnotetext{
Address for correspondence Celeste Durnwald, MD, Maternal and Child Health Center, University of Pennsylvania, 3400 Spruce Street, Philadelphia, PA 19104 celeste.durnwald@uphs.upenn.edu.

Note

This study was presented at the 37th Annual Meeting of the Society for Maternal Fetal Medicine, Las Vegas, NV, January 25-27, 2017.

Clinical Trials Registration

The clinical trial was registered at https://clinicaltrials.gov/, NCT02128997.

Conflict of Interest

None.
} 


\section{Keywords}

cesarean delivery; negative pressure wound therapy; obese; surgical site infection; wound complication

The incidence of surgical site infections (SSIs) after cesarean delivery has decreased in recent years, likely as a result of interventions aimed at reducing infection such as the administration of antibiotics prior to skin incision and the use of more effective skin preparation agents. ${ }^{1-3}$ However, the cost of infection remains significant from both financial and social perspectives. An infection after a cesarean delivery is estimated to cost more than $\$ 4,700$, and the toll of the morbidity of an infection on a newly postpartum woman and her infant cannot be discounted. ${ }^{4}$ Obese women, in particular, are two times more likely than normal weight women to develop a SSI after a cesarean delivery. ${ }^{1,5}$

Negative pressure wound therapy (NPWT) is routinely used to improve wound healing after the development of an infection and recently has been introduced as a prophylactic wound dressing to prevent infection. ${ }^{6}$ NPWT is a method in which a vacuum device is placed on the wound to remove excess interstitial fluid, increase tissue vascularity, decrease bacterial colonization with a layer of silver in the interface layer of the fabric, and place mechanical stress on adjacent wound edges which aids in wound contractility and closure. It has been utilized in high-risk orthopaedic and cardiothoracic surgical patient populations to significantly decrease the incidence of SSIs. ${ }^{6-8}$ Although the device has been shown to be associated with reduced wound infection after a cesarean delivery, the studies are all retrospective, leading to a concern for selection bias. ${ }^{9,10}$ The absence of a randomized trial renders estimates of SSI rates in this population uncertain at best.

Therefore, the objective of our study was to perform a randomized controlled feasibility trial, investigating the effect of the prophylactic NPWT system versus standard wound care (WC) on the development of a postoperative SSI and/or wound dehiscence, to yield accurate estimates of SSI rates, and to inform sample size calculations for a larger trial.

\section{Study Design}

This was a randomized controlled feasibility trial performed from May 2014 through March 2016 at a tertiary care university medical center. Women $\geq 18$ years old with an initial body mass index (BMI) $\geq 30 \mathrm{~kg} / \mathrm{m}^{2}$ at their first prenatal visit $<22$ weeks who presented in labor (defined as regular contractions with cervical change)were eligible for enrollment. Exclusion criteria included initiation of prenatal care after 23 weeks (no early BMI), chronic steroid use, pregestational diabetes, treatment for an active malignancy, an allergy to silver (contraindication to Prevena NPWT), scheduled cesarean section, and planned vertical skin incision. Women were consented in labor upon admission, prior to clinician decision for cesarean delivery so that management of the labor process was not modified due to participation. The study was approved by the University of Pennsylvania Institutional Review Board and was registered at clinicaltrials.gov (identifier NCT02128997). 
Once decision for cesarean delivery was established, randomization was performed using a computer-generated randomization scheme (Research Electronic Data Capture [REDCap]).

${ }^{11}$ Study protocol mandated that all skin incisions were closed using staples; however, all other surgical management was at the discretion of the obstetric provider. Additional standard interventions in place at our institution to prevent SSI and wound disruption include preoperative antibiotics, the use of Sage $2 \%$ chlorhexidine gluconate surgical preparation cloths for women in labor for longer than 6 hours (Sage Products, Cary, IL), the use of a vaginal prep (instituted April 2015) in all laboring women prior to cesarean and the placement of subcutaneous sutures prior to skin closure in all obese women. Preoperative antibiotics included weight-based dosing of cefazolin $2 \mathrm{~g}$ intravenous (IV) (weight $<120 \mathrm{~kg}$ ) or $3 \mathrm{~g} \mathrm{IV} \mathrm{(weight} \geq 120 \mathrm{~kg}$ ). If a patient has a $\beta$ lactam allergy, clindamycin $900 \mathrm{mg}$ IV and gentamicin $4 \mathrm{mg} / \mathrm{kg}$ IV based on actual body weight (max dose $500 \mathrm{mg}$ ) was used. All preoperative antibiotics were administered prior to skin incision. Chlorhexidine gluconate surgical preparation cloths were used to clean the maternal abdomen prior to surgical scrub in all women who have been in labor for longer than 6 hours. In addition, all women received an abdominal surgical prep of DuraPrep (if maternal and fetal well-being was stable for the 3-minute wait period for drying) or chlorhexidine if plan was for draping and surgical incision prior to the 3-minute drying period.

Patients randomized to receive the intervention had a Prevena Incision Management System (Acelity, San Antonio, TX) placed after the surgeons closed the skin incision under sterile condition prior to removal of surgical drapes (Fig. 1). All providers who placed the device participated in a training session about the use of the device from study personnel according to the Acelity company on-site training. Standard WC included placement of a Telfa bandage overlaid with a $4 \mathrm{~cm} \times 4 \mathrm{~cm}$ gauze pad and surgical tape. As per hospital protocol, standard WC dressings were removed 24 hours after the completion of surgery. The NPWT Prevena system remained in place until postoperative day 3 when study personnel removed it. On postoperative day 2, all patients were administered a questionnaire reviewing their pain, breastfeeding status, and activities of daily living. Complications with the Prevena NPWT system including device troubleshooting and/or decision for device removal were made by the primary investigators (K.E.R. and C.P.D.). On postoperative day 3, study personnel removed the patient's staples and a photo of the incision was taken prior to discharge.

Patients were contacted 2 weeks after discharge to inquire about wound healing, and schedule a 4-week postsurgical study visit. At the follow-up visit, each patient was asked about any wound complications during the 4 weeks postpartum and her chart was reviewed for any office or emergency department visits or hospital admissions related to wound complications.

The primary outcome was a composite outcome of a SSI, incision dehiscence, or wound opening by a provider that required packing during the 4-week postsurgical period. A SSI was defined as erythema and/or purulent drainage with or without fever that required antibiotic therapy. An incision dehiscence or wound opening included any disruption of the skin closure with subcutaneous tissue exposure or a wound opening that required packing. Secondary outcomes included sharp and tingling pain scores (range: $0-10$ ), difficulty with 
activities of daily living on postoperative day 2 (range: 0 for "no difficulty" to 4 "so much difficulty I could not do it"), the development of a skin blister at the site of the dressing, postpartum length of stay, an office or emergency room visit during the 4-week period due to a wound concern, or a patient-reported incisional concern at the 2-week study phone call.

Study personnel collected all outcomes and clinical characteristics by individual chart review of each woman's electronic medical records. Study data were collected and managed using REDCap tools hosted at the University of Pennsylvania. ${ }^{11}$

A sample size calculation was performed assuming a rate of $10 \%$ for the primary outcome in the control group (based on prior studies in obese women) and a 5\% complication rate in the intervention group, $90 \%$ power, and an a of 0.05 . Based on this calculation, 621 patients would be required in each arm of the study. To perform a feasibility study of sufficient size to estimate the true rate of the outcome, an a priori enrollment goal of $10 \%$ of the calculated sample size was selected with the additional allowance of $10 \%$ loss to follow-up, which resulted in a sample size of 68 patients per arm. ${ }^{12}$

Continuous variables were compared with $t$-test or Wilcoxon rank sum where appropriate. Categorical variables were compared with Fisher's exact test. All analyses were completed as intention to treat. Finally, we calculated the number needed to treat with NPWT to reduce one surgical site complication and compare the associated costs of use of NPWT versus those associated with treatment of SSIs after a cesarean.

\section{Results}

During the study period, 970 women met eligibility criteria and $480(49.4 \%)$ consented to participate. Of the 480 women who consented, 136 (28\%) underwent cesarean delivery and were randomized: 69 to standard WC and 67 to NPWT (Fig. 2). Seventeen patients were lost to follow-up during the 4-week period, leaving 58 in the standard WC group and 61 in the NPWT group for analysis.

Baseline clinical and labor characteristics were similar between the two treatment groups (Table 1). In addition, there were no significant differences in the surgical characteristics between the groups (Table 2). The majority of women had a cesarean delivery performed for nonreassuring fetal heart tracing (38.6\%) or arrest of dilation (28.6\%) and received an epidural for anesthesia (88.2\%). In general, surgical time was $\sim 60$ minutes and most women had less than a liter of blood loss. Additional standard interventions to prevent SSI and wound disruption such as preoperative antibiotics, chlorhexidine cloth use, and vaginal and abdominal preps were similar between the groups.

Table 3 summarizes the primary and secondary outcomes of our study. The composite wound outcome was reduced among women in the NPWT group (4.9 vs. 6.9\%), but this difference did not reach statistical significance $(p=0.71)$. Pain scores, postpartum length of stay, and reports of wound concerns at the week 2 call were all similar between women receiving standard WC and NPWT. 
Although there was no statistically significant difference, four times as many women who received the NPWT system had a skin blister after removal of the device compared with standard WC (13.1\% NPWT vs. 3.6\% standard WC, $p=0.10)$. None of the women with skin blistering required additional treatment. There were relatively few other complications with the NPWT devices. Five NPWT systems had malfunctions with either suction or the device turning off during the 3-day postoperative study period, but only one device ultimately required removal. Four patients reported discomfort with the device, but only one requested the device be removed due to the discomfort. One patient needed to have the canister on the NPWT system replaced due to excessive fluid removal from the incision that surpassed the canister capacity.

In considering the cost of the intervention, our findings demonstrate that 28 NPWT systems would need to be placed to prevent one SSI in an obese woman undergoing an unscheduled cesarean delivery. At a per device cost of $\$ 544$, prevention of a single infection would cost approximately $\$ 15,000$. Thus, the prevention of one SSI after a cesarean delivery would increase postsurgical health care costs, an additional $\$ 10,300$ beyond the average cost attributed to the infection itself.

\section{Conclusion}

Our study is the first randomized controlled feasibility trial to determine the rate of surgical site complications after cesarean delivery in obese laboring women using a NPWT device compared with routine postoperative WC. All previous studies investigating the use of the NPWT system after cesarean delivery have been retrospective, and therefore, limited by the potential for selection bias and confounding by maternal comorbidities. ${ }^{9,10}$

Our results show that the rate of wound complication, even in this high-risk population, is much lower than most previously reported with only $6.9 \%$ of women in the routine treatment group and $4.9 \%$ of women in the NPWT having clinically significant wound complications. The overall low rate of infection in our study (5.9\%) is consistent with a 2015 randomized trial investigating the use of different antiseptic agents prior to cesarean delivery and the development of SSI. ${ }^{3}$ At many institutions, including our own, additional infection prevention measures are routinely utilized including weight-based preoperative antibiotics, use of Sage cloths in labor, vaginal prep prior to cesarean, and closure of the subcutaneous tissue in obese women. ${ }^{9,10}$ As a result of these risk reduction efforts, contemporary infection rates may be lower than prior published results. Given the overall lower rate of wound morbidity, the prophylactic use of a NPWT system may provide little additional benefit to decrease the risk of wound complications. Differences in baseline wound complication rates may also explain why the use of NPWT has previously shown considerable benefit in decreasing postoperative wound complications in other surgical setting, such as cardiothoracic and orthopaedic surgeries, which have higher infection rates ( 24-30\%).

Furthermore, our results revealed that, even in the highest risk group of patients, NPWT only reduced surgical site complications by $2 \%$. Given the incidence of the complications and the effect size, the revised sample size calculations indicate that 4,356 women would need to be randomized to perform an appropriately powered study. Given that only $28 \%$ of consented 
patients in our study underwent cesarean, a total of 15,557 women would need to be consented to achieve the specified sample size for randomization. This would certainly require a multicenter design as our study took 22 months to enroll 136 women on a labor and delivery unit with $\sim 4,000$ deliveries per year. Ultimately, the revised sample size was more than three times higher than our preliminary calculations, which underscores the potential value of conducting a randomized controlled feasibility study.

In a 2015 editorial response to a decision analysis evaluating the cost effectiveness of a NPWT system after cesarean delivery, it was stated, "The question that needs to be asked first is whether negative pressure therapy has any efficacy...does it reduce the rate of surgical site infections...."13 Although our study does not meet the editorial's call for an "adequately powered randomized trial," our results indicate that such a study would need to be extremely large, very expensive, and may show little to no benefit of placement of a NPWT system at the time of cesarean delivery compared with other risk reduction strategies that are already in place.

In conclusion, our study provides important information that can be used to design and complete an appropriately powered trial. To continue to improve care and reduce infections in obese women, adequately powered studies based on accurate estimates of the primary outcome are needed. Our study was able to provide important information to continue to work toward this goal. Thus, until an appropriately powered, large-scale randomized trial can be completed, and routine use of a prophylactic NPWT system may not be warranted.

\section{Acknowledgments}

\section{Funding}

This study was supported by the National Institute of Health Reproductive Epidemiology Training Gran (T32HD007440). All study devices were provided by Acelity. The funding sources had no role in study design, data collection, or analysis.

\section{References}

1. Krieger Y, Walfisch A, Sheiner E. Surgical site infection following cesarean deliveries: trends and risk factors. J Matern Fetal Neonatal Med. 2017; 30(01):8-12. [PubMed: 27023698]

2. Smaill FM, Grivell RM. Antibiotic prophylaxis versus no prophylaxis for preventing infection after cesarean section. Cochrane Database Syst Rev. 2014; 10:CD007482.

3. Tuuli MG, Liu J, Stout MJ, et al. A randomized trial comparing skin antiseptic agents at cesarean delivery. N Engl J Med. 2016; 374(07):647-655. [PubMed: 26844840]

4. Olsen MA, Butler AM, Willers DM, Gross GA, Hamilton BH, Fraser VJ. Attributable costs of surgical site infection and endometritis after low transverse cesarean delivery. Infect Control Hosp Epidemiol. 2010; 31(03):276-282. [PubMed: 20102279]

5. Kominiarek MA, Vanveldhuisen P, Hibbard J, et al. Consortium on Safe Labor. The maternal body mass index: a strong association with delivery route. Am J Obstet Gynecol. 2010; 203(03):264.e1264.e7. [PubMed: 20673867]

6. Huang C, Leavitt T, Bayer LR, Orgill DP. Effect of negative pressure wound therapy on wound healing. Curr Probl Surg. 2014; 51(07):301-331. [PubMed: 24935079]

7. Stannard JP, Volgas DA, McGwin G III, et al. Incisional negative pressure wound therapy after highrisk lower extremity fractures. J Orthop Trauma. 2012; 26(01):37-42. [PubMed: 21804414] 
8. Grauhan O, Navasardyan A, Hofmann M, Müller P, Stein J, Hetzer R. Prevention of poststernotomy wound infections in obese patients by negative pressure wound therapy. J Thorac Cardiovasc Surg. 2013; 145(05):1387-1392. [PubMed: 23111014]

9. Anglim B, O’Connor H, Daly S. Prevena ${ }^{\mathrm{TM}}$, negative pressure wound therapy applied to closed Pfannenstiel incisions at time of caesarean section in patients deemed at high risk for wound infection. J Obstet Gynaecol. 2015; 35(03):255-258. [PubMed: 25383909]

10. Swift SH, Zimmerman MB, Hardy-Fairbanks AJ. Effect of single-use negative pressure wound therapy on postcesarean infections and wound complications for high-risk patients. J Reprod Med. 2015; 60(5-6):211-218. [PubMed: 26126306]

11. Harris PA, Taylor R, Thielke R, Payne J, Gonzalez N, Conde JG. Research Electronic Data Capture (REDCap)-a metadata-driven methodology and workflow process for providing translational research informatics support. J Biomed Inform. 2009; 42(02):377-381. [PubMed: 18929686]

12. Connelly LM. Pilot studies. Medsurg Nurs. 2008; 17(06):411-412. [PubMed: 19248407]

13. Rouse DJ. Prophylactic negative pressure wound therapy: we need proof before application. Obstet Gynecol. 2015; 125(02):297-298. [PubMed: 25569011] 
Fig. 1.

Prevena negative wound pressure therapy system. 


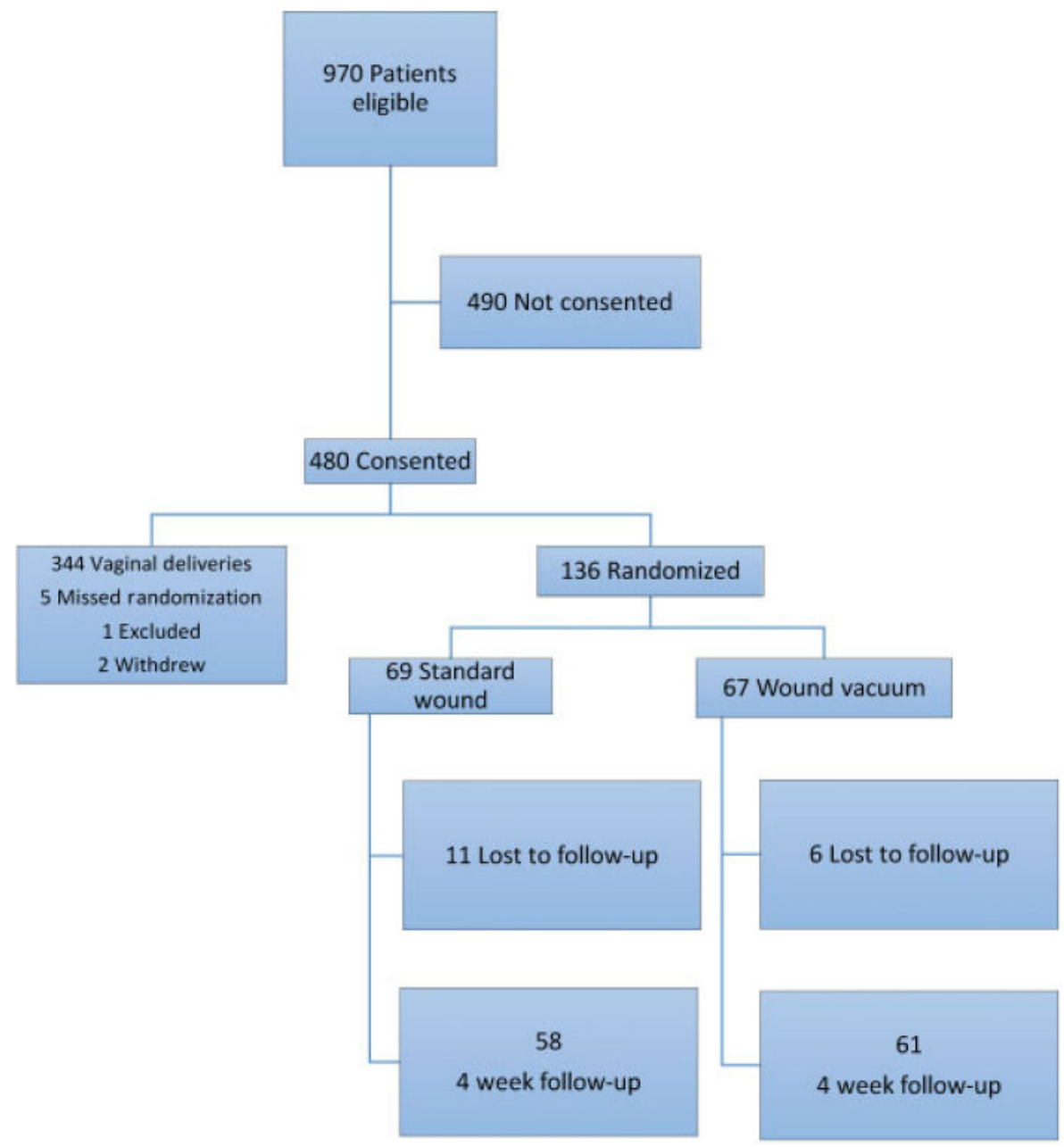

Fig. 2.

Study enrollment. 
Table 1

Clinical and labor characteristics of the study population

\begin{tabular}{|c|c|c|c|}
\hline Characteristic & Standard wound care $(n=58) n(\%)$ & NPWT $(n=61) n(\%)$ & $p$-Value \\
\hline Age, $y^{a}$ & $29(24-34)$ & $27(24-32)$ & 0.34 \\
\hline \multicolumn{4}{|l|}{ Race } \\
\hline Black & 45 (77.6) & $46(75.4)$ & \multirow[t]{4}{*}{0.87} \\
\hline White & $12(20.7)$ & $14(23.0)$ & \\
\hline Asian & $1(1.7)$ & 0 & \\
\hline Hawaiian/Pacific Islander & 0 & $1(1.6)$ & \\
\hline \multicolumn{4}{|l|}{ Insurance } \\
\hline Private & $23(39.7)$ & $20(32.8)$ & \multirow[t]{4}{*}{0.77} \\
\hline Medicaid & $32(55.2)$ & $39(63.9)$ & \\
\hline Medicare & $1(1.7)$ & $1(1.6)$ & \\
\hline Unknown & $2(3.5)$ & $1(1.6)$ & \\
\hline Nulliparous & $23(39.7)$ & $23(37.7)$ & 0.85 \\
\hline BMI, initial prenatal visit $\left(\mathrm{kg} / \mathrm{m}^{2}\right)^{a}$ & $35.1(32.6-42.1)$ & $36.1(33.2-41.8)$ & 0.60 \\
\hline Tobacco use & $3(5.2)$ & $5(8.2)$ & 0.72 \\
\hline Chronic hypertension & $7(12.1)$ & $9(14.8)$ & 0.79 \\
\hline Gestational diabetes & $4(7.0)$ & $5(8.2)$ & 0.99 \\
\hline History of cesarean delivery & $11(19.0)$ & $11(18.0)$ & 0.99 \\
\hline History of vaginal delivery & $20(30.5)$ & $19(31.1)$ & 0.85 \\
\hline Gestational age at delivery, $\mathrm{wk}^{a}$ & $39(38-40)$ & $39(38-40)$ & 0.45 \\
\hline Induction of labor & 44 (75.9) & $44(72.1)$ & 0.68 \\
\hline Length of labor, $\mathrm{h}^{a}$ & $18.5(11.2-26.7)$ & $22.3(12.4-27.6)$ & 0.53 \\
\hline Chorioamnionitis & $7(12.1)$ & $10(16.4)$ & 0.60 \\
\hline
\end{tabular}

Abbreviations: BMI, body mass index; NPWT, negative pressure wound therapy.

${ }^{a}$ Data are presented as median (interquartile range). 
Table 2

\section{Surgical characteristics}

\begin{tabular}{|c|c|c|c|}
\hline Characteristic & Standard wound care $(n=58) n(\%)$ & NPWT $(n=61) n(\%)$ & $p$-Value \\
\hline Preoperative antibiotics & $55(94.8)$ & $57(93.4)$ & 0.99 \\
\hline \multicolumn{4}{|l|}{ Indication for cesarean } \\
\hline Arrest of descent & $3(5.2)$ & $4(6.6)$ & \multirow[t]{6}{*}{0.62} \\
\hline Arrest of dilation & $19(32.8)$ & $15(24.6)$ & \\
\hline Fetal intolerance of labor & $23(39.7)$ & $23(37.7)$ & \\
\hline Failed IOL & $9(15.5)$ & $15(24.6)$ & \\
\hline Fetal malpresentation & $2(3.4)$ & $4(6.6)$ & \\
\hline Elective $^{a}$ & $1(1.7)$ & 0 & \\
\hline TOLAC & $11(19.0)$ & $11(18.0)$ & 0.99 \\
\hline Sage prep & $47(88.7)$ & $47(87.0)$ & 0.99 \\
\hline \multicolumn{4}{|l|}{ Abdominal prep } \\
\hline DuraPrep & $31(56.4)$ & $43(70.5)$ & \multirow[t]{2}{*}{0.13} \\
\hline Chlorhexidine & $24(43.6)$ & $18(29.5)$ & \\
\hline \multicolumn{4}{|l|}{ Anesthesia } \\
\hline Epidural & $52(89.7)$ & $53(86.9)$ & \multirow[t]{3}{*}{0.99} \\
\hline Spinal & $2(3.4)$ & $3(4.9)$ & \\
\hline General endotracheal anesthesia & $4(6.9)$ & $5(8.2)$ & \\
\hline Length of surgery, $\min ^{b}$ & $62(52-70)$ & $61(50-72)$ & 0.95 \\
\hline Blood loss, $\mathrm{mL}^{b}$ & $838(800-1,000)$ & $900(800-1,000)$ & 0.90 \\
\hline Vaginal hand & $2(3.5)$ & $1(1.6)$ & 0.61 \\
\hline
\end{tabular}

Abbreviations: IOL, induction of labor; NPWT, negative pressure wound therapy; TOLAC, trial of labor after cesarean.

a Patient with a history of prior cesarean initially decided to attempt a trial of labor, reached $4 \mathrm{~cm}$ and then decided to undergo repeat cesarean delivery by choice.

$b_{\text {Data are presented as median (interquartile range). }}$ 
Table 3

Incidence of primary and secondary outcomes in study population

\begin{tabular}{|c|c|c|c|}
\hline & Standard wound care $(n=58) n(\%)$ & NPWT $(n=61) n(\%)$ & $p$-Value \\
\hline \multicolumn{4}{|l|}{ Primary outcomes } \\
\hline Composite wound outcome ${ }^{a}$ & $4(6.9)$ & $3(4.9)$ & 0.71 \\
\hline Wound infection & $4(6.9)$ & $2(3.3)$ & 0.44 \\
\hline Wound requiring opening/packing & $2(3.5)$ & $3(4.9)$ & 0.99 \\
\hline \multicolumn{4}{|l|}{ Secondary outcomes } \\
\hline Skin blister & $2(3.6)$ & $8(13.1)$ & 0.10 \\
\hline POD 2 sharp pain $(0-10)$ & $5.5(3-8)^{b}$ & $6(4-8)^{b}$ & 0.56 \\
\hline POD 2 tingling pain $(0-10)$ & $1.5(0-6)^{b}$ & $2(0-6)^{b}$ & 0.99 \\
\hline Postpartum length of stay, $d$ & $3(3-4)^{b}$ & $3(3-4)^{b}$ & 0.78 \\
\hline Wound concern reported at week 2 call & $11(20.0)$ & $12(21.8)$ & 0.99 \\
\hline
\end{tabular}

Abbreviations: NPWT, negative pressure wound therapy; POD, postoperative day.

${ }^{a}$ Composite wound outcome was composed of surgical site infections or those which required opening and/or repacking.

$b_{\text {Data are presented as median (interquartile range). }}$ 